\title{
GABA A receptor $\pi$ subunit promotes apoptosis of HTR-8/SVneo trophoblastic cells: Implications in preeclampsia
}

\author{
JUNJIE LU, QIAN ZHANG, DONGMEI TAN, WENPING LUO, \\ HAI ZHAO, JING MA, HAO LIANG and YI TAN \\ Laboratory Animal Center, Chongqing Medical University, Yuzhong, Chongqing 400016, P.R. China
}

Received May 18, 2015; Accepted May 10, 2016

DOI: $10.3892 / \mathrm{ijmm} .2016 .2608$

\begin{abstract}
Gamma-aminobutyric acid (GABA) functions primarily as an inhibitory neurotransmitter through its receptors in the mature central nervous system. The GABA type A receptor $\pi$ subunit (GABRP) has been identified in the tissues of the reproductive system, particularly in the uterus. In addition, we have previously detected GABRP expression in both human and mouse placentas. To examine the role of GABRP in trophoblastic cell invasion, we constructed a pIRES2-GABRP-EGFP plasmid which was used for the transfection of a human placental cell line derived from first trimester extravillous trophoblasts (HTR-8/SVneo). The number of invaded cells was decreased by GABRP overexpression. Notably, the decrease in the invasive cell number may be due to the increased apoptosis of the HTR-8/SVneo cells following GABRP transfection, which was further confirmed by flow cytometry, reverse transcriptionquantitative polymerase chain reaction (RT-qPCR) and western blot analysis. Based on the increased apoptosis of trophoblastic cells in pregnancies complicated by preeclampsia (PE) and the fact that GABRP promotes the apoptosis of trophoblastic cells, we hypothesized that GABRP expression is increased in the placental tissues from patients with PE compared with that in the normal groups and this hypothesis was confirmed by RT-qPCR and immunohistochemical analysis. Taken together, these findings imply that GABRP plays an important role in placentation and this pathway may be a promising molecular target for the development of novel therapeutic strategies for PE.
\end{abstract}

\section{Introduction}

Gamma-aminobutyric acid (GABA) acts mainly as an inhibitory neurotransmitter at GABA type A (GABA A)

Correspondence to: Professor Yi Tan, Laboratory Animal Center, Chongqing Medical University, 1 Medical School Road, Yuzhong, Chongqing 400016, P.R. China

E-mail: tanyee66@hotmail.com

Key words: gamma-aminobutyric acid type A receptor $\pi$ subunit, trophoblast, apoptosis, preeclampsia receptors to mediate the synaptic inhibition of neuronal activity in the mammalian brain (1). The GABA A receptor is a heteropentamer, consisting of five subunits that form a chloride ion channel. In addition to being located on central neurons, functional GABA A receptors, particularly the GABA A receptor $\pi$ subunit (GABRP), have also been found in peripheral tissues, such as the uterus, prostate, ovaries, placenta, gall bladder, lung and small intestine $(2,3)$. It has been reported that the assembly of GABRP into a recombinant GABA A receptor may change the sensitivity of the receptor to modulatory agents such as GABA and allopregnanolone (4). In addition, due to its upregulation in the human endometrium during the window of implantation, GABRP may play an important role in the generation of a receptive endometrium (5). In our previous study, GABRP was detected in both human and mouse placenta; however, the precise function of GABRP in trophoblastic cells during placentation remains poorly defined (7).

In the present study, we report that GABRP overexpression may result in the decreased invasion of HTR-8/SVneo cells, possibly induced by elevated apoptosis. It has been suggested that the elevated apoptosis of cytotrophoblasts (CTBs) in preeclampsia $(\mathrm{PE})$ contributes to the placental dysfunction, which is characteristic of this disorder (6). Given the role of GABRP in enhancing the apoptosis of HTR-8/SVneo trophoblastic cells, we hypothesized that there may be a correlation between GABRP expression and PE. As expected, the results of reverse transcription-quantitative polymerase chain reaction (RT-qPCR) and immunohistochemical analysis revealed that the expression of GABRP was increased in placental tissues from patients with PE compared with that in the normal controls, which may contribute to the shallow trophoblastic invasion and hence, result in the onset of PE. In our laboratory, we have previously demonstrated that the GABRP protein level was decreased in cultured mouse stromal cells during the decidualization process, and that GABA suppressed this procress through GABA A receptors, which suggests that GABRP impaired the remodeling of the decidua (7). Based on these results, we concluded that GABRP may adversely affect placental function complicated with PE through intrinsic (trophoblastic) factors in combination with extrinsic (maternal uterine) factors. Further studies are warranted to determine whether GABRP has the potential to be used as a biomarker or a therapeutic molecular target for PE. 


\section{Materials and methods}

Human placental tissue samples. Six placentas from patients with PE and six placentas from normal pregnancies were obtained from patients at the First Affiliated Hospital of Chongqing Medical University (Chongqing, China). The criteria for the diagnosis of severe PE were strictly based on those of the American Congress of Obstetricians and Gynecologists (2002). The study was approved by the Ethics Committee of Chongqing Medical University (Chongqing, China) and informed consent was obtained from all patients. The tissue samples were fixed in buffered formalin $(4 \%, \mathrm{~m} / \mathrm{v})$ and embedded in paraffin according to standard procedures.

Immunohistochemistry (IHC) and immumocytochemistry (ICC). IHC was performed with a biotin-streptavidin-peroxidase (SP; Zhongshan Goldenbridge, Beijing, China) and 3,3'-diaminobenzidine (DAB; Zhongshan Goldenbridge). The sections $(5 \mathrm{~mm})$ were deparaffinized and rehydrated in xylene and ethanol gradients. The slides were boiled in citrate buffer $(10 \mathrm{mM}$ citrate sodium, $10 \mathrm{mM}$ citric acid, $\mathrm{pH}$ 6.0) in a microwave oven at $92-98^{\circ} \mathrm{C}$ for $15 \mathrm{~min}$ for antigen retrieval. The sections were then sequentially incubated with $3 \% \mathrm{H}_{2} \mathrm{O}_{2}$ in methanol for $10 \mathrm{~min}$ to quench endogenous peroxidase, and in normal goat serum as the blocking solution for $20 \mathrm{~min}$. The sections were incubated with primary antibodies against GABRP (ab26055; Abcam, Cambridge, UK) or rabbit $\mathrm{IgG}$ isotypic control at $4^{\circ} \mathrm{C}$ overnight and biotinylated secondary antibody. The sections were then incubated in HRP-streptavidin (Zhongshan Goldenbridge) for $30 \mathrm{~min}$ at room temperature and then detected using the chromogenic peroxidase substrate, $\mathrm{DAB}$, and counterstained with hematoxylin.

For ICC staining, the HTR-8/SVneo trophoblastic cells (obtained from Professor H. Wang, Institute of Zoology, Chinese Academy of Sciences, Beijing, China) were cultured on coverslips for $48 \mathrm{~h}$. The coverslips were fixed in $4 \%$ paraformaldehyde for $20 \mathrm{~min}$ at room temperature, washed in phosphate-buffered saline (PBS) and permeabilized for $10 \mathrm{~min}$ with $0.25 \%$ Triton-100 in PBS. The cells were then incubated with $1 \%$ bovine serum albumin (BSA) in PBS/Tween 20 (PBST) for $30 \mathrm{~min}$ to block the non-specific binding of the antibodies. The cells were incubated with primary antibody or rabbit IgG isotype control overnight at $4^{\circ} \mathrm{C}$, and then incubated with a peroxidase-conjugated secondary antibody for $60 \mathrm{~min}$ at $37^{\circ} \mathrm{C}$. The slides were stained with DAB and counterstained with hematoxylin.

Cell culture and treatment. An immortalized, first trimester extravillous trophoblast (EVT) cell line, HTR-8/SVneo, which was derived from a short-lived, primary EVT cell line, was used in the present study. The HTR-8/SVneo cells were maintained in RPMI-1640 medium, supplemented with $10 \%$ heat-inactivated fetal bovine serum (FBS; Gibco-BRL, Carlsbad, CA, USA), $100 \mathrm{U} / \mathrm{ml}$ penicillin (Beyotime Biotech, Jiangsu, China) and $100 \mu \mathrm{g} / \mathrm{ml}$ streptomycin (Beyotime Biotech) under standard culture conditions $\left(37^{\circ} \mathrm{C}\right.$ in a $5 \%$ humidified $\mathrm{CO}_{2}$ incubator). The cells were transiently transfected with full-length GABRP after being seeded onto $35-\mathrm{mm}$ dishes overnight. In order to exogenously express GABRP, the GABRP sequence was sub-cloned into the pIRES2-EGFP plasmid vector. After the GABRP sequence was inserted into the vector, sequencing analysis was conducted to ensure that this vector was capable of specifically expressing GABRP (constructed by Invitrogen, Carlsbad, CA, USA).

Matrigel invasion assay. An invasion assay was performed in Matrigel-coated (BD Biosciences, Bedford, MA, USA) Transwell inserts ( $6.5 \mathrm{~mm}$; Costar, Cambridge, UK) containing polycarbonate filters with a pore size of $8 \mu \mathrm{m}$. Briefly, the inserts were pre-coated with $100 \mu$ l Matrigel matrix $(1 \mathrm{mg} / \mathrm{ml})$ at $37^{\circ} \mathrm{C}$ for $4 \mathrm{~h}$ for gelling. The HTR-8/SVneo cells $\left(1 \times 10^{5}\right.$ cells) in $200 \mu \mathrm{l}$ serum-free medium were plated in the upper chamber, whereas medium with $10 \%$ FBS was added to the lower well. After incubating for $24 \mathrm{~h}$, the cells on the Matrigel side of the insert were scraped by a cotton swab. The inserts were then fixed in methanol for $10 \mathrm{~min}$ at room temperature and stained with hematoxylin and eosin (H\&E; Zhongshan Goldenbridge). The cells which had invaded to the other side of the insert were counted under a light microscope (IX51; Olympus, Tokyo, Japan) in ten random fields at magnification of $x 200$. The assay was repeated three times, and the results are represented as the percentage means of invasion \pm standard deviation (SD) compared with the control.

Western blot analysis. The HTR-8/SVneo cells transfected with overexpressing plasmid groups were lysed for protein extraction using RIPA supplemented with phenylmethylsulfonyl fluoride (PMSF) (both from Beyotime Biotech). The Bradford assay (Beyotime Biotech) was used to determine the protein concentration of each sample. Samples containing 50-100 $\mu \mathrm{g}$ of extracted protein were separated by $10 \%$ sodium dodecyl sulfate-polyacrylamide gel electrophoresis (SDS-PAGE). The separated proteins were transferred to $0.22 \mu \mathrm{m}$ nitrocellulose membranes and were incubated with primary antibodies against GABRP (ab26055; Abcam); caspase-3 (19677-1-AP; ProteinTech Group, Wuhan, China); cleaved caspase-3 (Asp175; Cell Signaling Technology, Inc., Danvers, MA, USA); Bcl-2 (AP13823c), Bad (AP1314c) and Bax (AP18517a) (all from Abgent, Inc., San Diego, CA, USA); and GAPDH (AP0063; Bioworld Technology, Inc., St. Louis Park, MN, USA) at 1:1,000 dilution. The secondary antibody was goat anti-rabbit IgG (1:1,000; Zhongshan Goldenbridge). All experiments were repeated at least three times.

Flow cytometry (FCM). The HTR-8/SVneo cells were transiently transfected with overexpressing (pIRES2-EGFP and pIRES2-GABRP-EGFP) plasmid groups and after $72 \mathrm{~h}$, they were harvested using trypsin without EDTA, washed with PBS, resuspended in $1 \mathrm{ml}$ binding buffer, and stained for 15 min with fluorescein isothiocyanate (FITC)-Annexin V and propidium iodide (PI) in the dark at room temperature, according to the manufacturer's instructions. Cell analysis was performed by a flow cytometer (FACScan) equipped with CellQuest software (both from BD Biosciences). The cells were sorted into living, necrotic, early apoptotic and late apoptotic cells. The relative ratio of early and late apoptotic cells was calculated for further comparison. This assay was repeated at least three times. 


\section{HTR-8/SVneo}
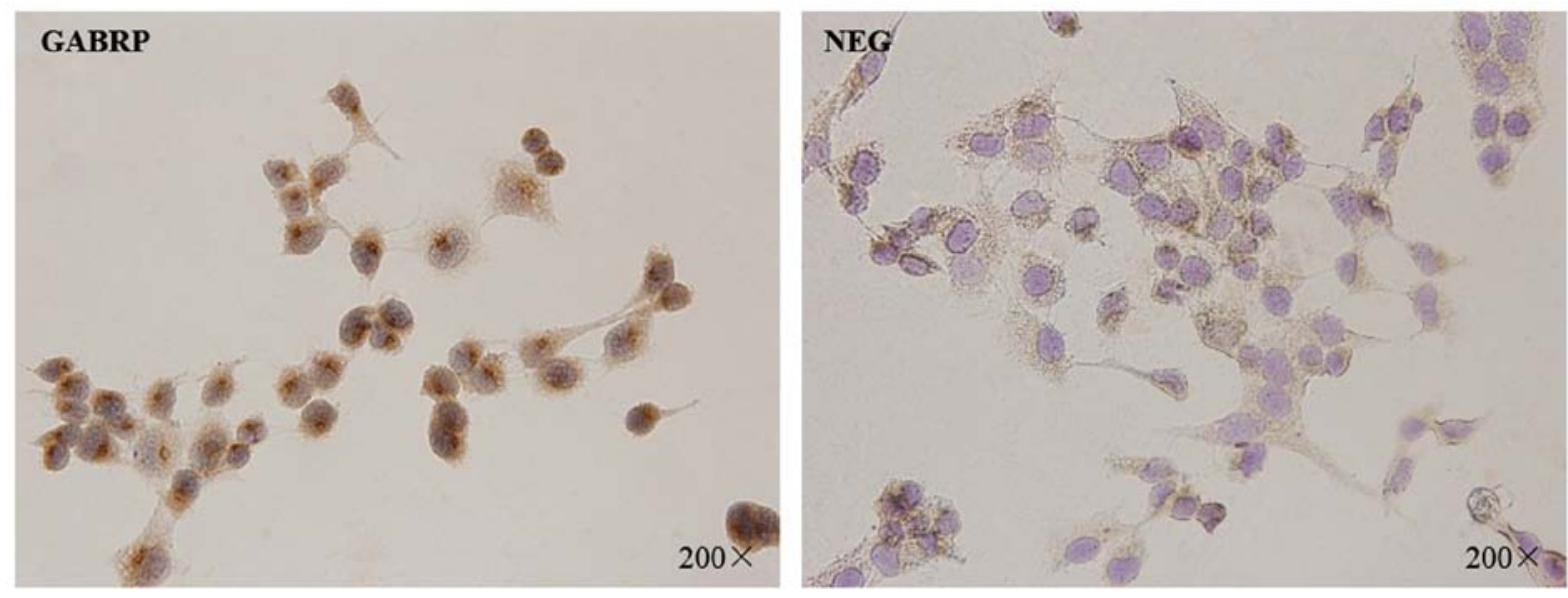

Figure 1. Immunolocalization of gamma-aminobutyric acid type A receptor $\pi$ subunit (GABRP) in HTR-8/SVneo cells. GABRP expression was detected in the cytoplasm and the nuclear membrane of the HTR-8/SVneo cells. NEG, negative controls in which normal IgG was used in place of primary antibody. Original magnification x200.

$R T-q P C R$. Total RNA extraction from the placental tissues and the HTR-8/SVneo cells was performed using TRIzol reagent (Invitrogen) according to the manufacturer's instructions, and quantities of RNA were determined using a spectrophotometer (Thermo Fisher Scientific, Inc., Waltham, MA, USA) at $260 \mathrm{~nm}$. Two micrograms of total RNA was reverse transcribed using SuperScript III Reverse Transcriptase (Invitrogen).

qPCR was performed using SYBR Premix Ex Taq II (RR820A; Takara Bio, Inc., Kusatsu, Japan) in a CFX96 ${ }^{\mathrm{TM}}$ Real-Time PCR detection system (Bio-Rad, Berkeley, CA, USA) and analyzed using the $\Delta \Delta \mathrm{Ct}$ method according to the following equation: $\Delta \mathrm{Ct}$ (test) $=\mathrm{Ct}$ (target, test) - $\mathrm{Ct}$ (ref, test); $\Delta \mathrm{Ct}$ (calibrator) $=\mathrm{Ct}$ (target, calibrator) - $\mathrm{Ct}$ (ref, calibrator); $\Delta \Delta \mathrm{Ct}=\Delta \mathrm{Ct}$ (test) $-\Delta \mathrm{Ct}$ (calibrator); $2^{-\Delta \Delta \mathrm{Ct}}=$ relative quantitative value. The following PCR primers were used: GABRP (NM_014211.2) sense, 5'-CGACCGTGTTATCAATGACC-3' and antisense, 5'-CCCCAAACACAAAGCTAAAGCA-3'; Bcl-2 (NM_000657.2) sense, 5'-TTGTTCAAACGGGATTC ACA-3' and antisense, 5'-GAGCAAGTGCAGCCACAATA-3'; Bax (NM_001291428.1) sense, 5'-GCTGGACATTGGACTTC CTC-3' and antisense, 5'-CTCAGCCCATCTTCTTCCAG-3'; Bad (NM_004322.3) sense, 5'-CCTCAGGCCTATGCA AAA-3' and antisense, 5'-AAACCCAAAACTTCCGA TGG-3'; and GAPDH (NM_002046.5) sense 5'-AGCCACAT CGCTCAGACAC-3' and antisense, 5'-TGGACTCCACGAC GTACTC-3'. This assay was repeated at least three times.

Cell viability assay. A Cell Counting Kit-8 (CCK-8; Beyotime Biotech) was employed in this experiment to quantitatively evaluate cell viability. Following 24, 48 and 72 h of plasmid transfection, a CCK- 8 assay was performed using the HTR-8/SVneo cells. Briefly, the HTR-8/SVneo cells were seeded at $0.5 \times 10^{4} /$ well in 96 -well plates. RPMI-1640 culture medium (100 $\mu \mathrm{l} /$ well) was added to $10 \mu \mathrm{l}$ CCK-8 reagent after 20,44 and $68 \mathrm{~h}$ and incubated at $37^{\circ} \mathrm{C}$ for a further $4 \mathrm{~h}$, to form water-soluble formazan. The absorbances at 450 and $630 \mathrm{~nm}$ (calibrated wave) were determined using a microplate reader (Multiskan MK33; Thermo Fisher Scientific, Inc.). RPMI1640 medium containing $10 \%$ CCK-8 was used as a control.
The untreated control was set at $100 \%$, and the treated samples were normalized to this value according to the following equation: survival rate $(\%)=$ optical density $(\mathrm{OD})$ of the treated cells - OD of blank control/OD of negative control - OD of blank control x 100 .

Statistical analysis. The bands from western blot analysis were quantified using ImageJ2x software (http://imagej.net/ Image 2 ). All data are presented as the means \pm SD and statistical analysis was performed using SPSS software (SPSS Inc., Chicago, IL, USA). Furthermore, $\mathrm{P}<0.05$ was considered to indicate a statistically significant difference.

\section{Results}

GABRP expression in HTR-8/SVneo cells. To assess the localization pattern of GABRP within the cells, immunocytochemical analysis was performed on the HTR-8/SVneo trophoblastic cells (Fig. 1). The presence of GABRP was identified by the specific brown-colored staining of the cytoplasm and the nuclear membrane of the HTR-8/SVneo cells. Notably, GABRP expression was mainly located on the apical side of the nuclear membrane which showed particular polarization to a certain extent.

GABRP overexpression significantly inhibits the invasion of HTR-8/SVneo cells. GABRP expression in the trophoblastic cell line may play a role in controlling trophoblastic cell invasion. Thus, we constructed a pIRES2-GABRP-EGFP plasmid and used Matrigel cell invasion models in the HTR-8/SVneo cells to verify this assumption. As shown in Fig. 2A, the exogenous expression of GABRP was evaluated by RT-qPCR and western blot analysis. The results demonstrated that the number of invading HTR-8/ SVneo cells overexpressing GABRP was decreased compared with that in the control groups $(\mathrm{P}<0.05)$ (Fig. 2B).

GABRP overexpression decreases cell viability and promotes the apoptosis of HTR-8/SVneo cells. To determine whether the observed changes in invasiveness were due to the effects 
A


Figure 2. Gamma-aminobutyric acid type A receptor $\pi$ subunit (GABRP) overexpression significantly inhibits the invasion of HTR-8/SVneo cells. (A) Confirmation of GABRP overexpression using RT-qPCR and western blot analysis. GAPDH was used as an internal control in RT-qPCR and a loading control in western blot analysis. (B) Representative images of filters containing invaded cells in Matrigel invasion assay. The statistical bar graphs show the summary of three independent experiments (t-test, ${ }^{*} \mathrm{P}<0.05$ ).

of GABRP on cell apoptosis and/or viability, we examined the effects of GABRP on the viability and apoptosis of trophoblastic cells. The HTR-8/SVneo cells transfected with the pIRES2-GABRP-EGFP plasmid were subjected to the CCK-8 assay and FCM, respectively. Compared with the control groups, the viability of the HTR-8/SVneo cells was decreased $(\mathrm{P}<0.05)$ (Fig. 3A) and the proportion of apoptotic HTR-8/SVneo cells was increased (Fig. 3B) by GABRP $72 \mathrm{~h}$ after transfection.

Members of the Bcl-2 family mediate increased apoptosis of HTR-8/SVneo cells through GABRP overexpression. As members of the Bcl-2 family are the key regulators of apoptosis, we evaluated the expression of three important members of the family, namely Bcl-2, Bad and Bax. The mRNA levels of pro-apoptotic Bad and Bax were upregulated after GABRP transfection $(\mathrm{P}<0.05)$ (Fig. 4A), which was consistent with the protein levels $(\mathrm{P}<0.05)$ (Fig. 4B). Unexpectedly, the mRNA and protein levels of anti-apoptotic Bcl-2 were also upregulated $(\mathrm{P}<0.05)$ (Fig. 4A and B). Further evaluation by western blot analysis revealed that GABRP overexpression increased
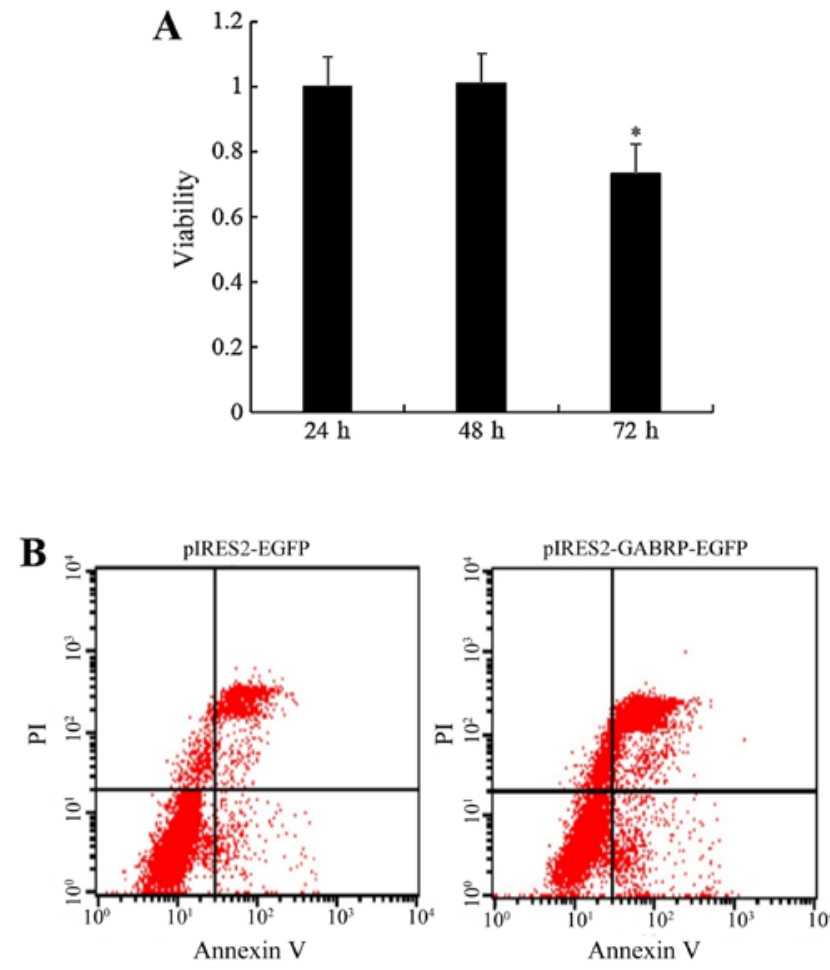

\begin{tabular}{ccc} 
Quad & Events & $\%$ Gated \\
\hline UL & 368 & 3.68 \\
UR & 1149 & 11.49 \\
LL & 8242 & 82.42 \\
LR & 241 & 2.41
\end{tabular}

\begin{tabular}{ccc} 
Quad & Events & $\%$ Gated \\
\hline UL & 736 & 7.36 \\
UR & 3401 & 34.01 \\
LL & 5304 & 53.04 \\
LR & 559 & 5.59
\end{tabular}

Figure 3. Effects of gamma-aminobutyric acid type A receptor $\pi$ subunit (GABRP) on cell viability and apoptosis of HTR-8/SVneo cells. (A) A CCK-8 assay was performed to demonstrate a decrease in cell viability of the HTR-8/SVneo cells 24, 48 and $72 \mathrm{~h}$ after GABRP transfection (t-test, ${ }^{*} \mathrm{P}<0.05$ ). (B) Flow cytometric analysis showed an increase in apoptosis of the HTR-8/SVneo cells $72 \mathrm{~h}$ after GABRP transfection. A representative image from three independent experiments is shown. UL, upper left panel (necrotic cells); UR, upper right panel (late apoptotic cells); LL, lower left panel (live cells); LR, lower right panel (early apoptotic cells).

the protein expression of cleaved caspase-3 (active form) and caspase-3 (inactive form) $(\mathrm{P}<0.05)$ (Fig. 4C).

GABRP is upregulated in placental villi from patients with $P E$. The apoptosis of villous trophoblasts is increased in PE and it is generally believed that PE is associated with impaired trophoblast invasion $(8,9)$. Thus, we hypothesized that GABRP may be involved in the onset of PE and we collected placental villi from patients with $\mathrm{PE}$ and from normal pregnancy controls of similar gestational stages for IHC analysis and RT-qPCR. Immunoreactivity for GABRP was consistently found in the villous syncytiotrophoblasts (STB) and CTBs of both groups. However, GABRP was strongly expressed in the PE group compared with the normal control group (Fig. 5A). Compared with the normal control group, the mRNA expression of GABRP was upregulated in the $\mathrm{PE}$ group $(\mathrm{P}<0.05)$ (Fig. 5B).

\section{Discussion}

Apoptosis is an essential feature of normal placental development; however, it may also be involved in the pathophysiology 

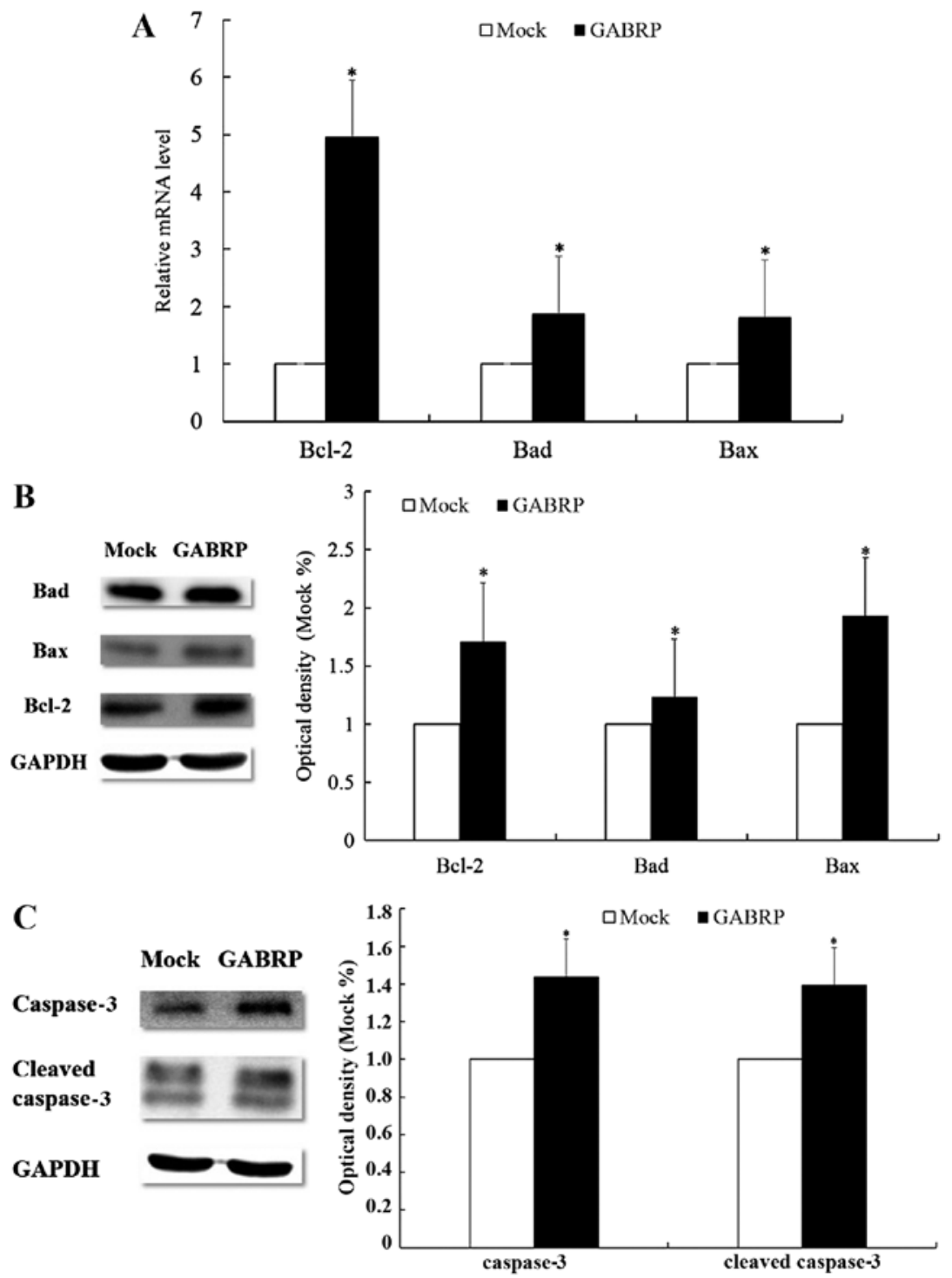

Figure 4. Regulators of HTR8/SVneo cell apoptosis following gamma-aminobutyric acid type A receptor $\pi$ subunit (GABRP) overexpression for $72 \mathrm{~h}$. (A) RT-qPCR revealed that the mRNA levels of the anti-apoptotic Bad and Bax and the pro-apoptotic Bcl-2 were increased following GABRP overexpression. (B) Western blot analysis showed that the anti-apoptotic Bad and Bax and pro-apoptotic Bcl-2 protein levels, were increased following GABRP overexpression. (C) Western blot analysis demonstrated that the effector caspase- 3 and its active form, cleaved caspase-3, were increased following GABRP overexpression. GAPDH was used as an internal control for RT-qPCR and as a loading control for western blot analysis. (t-test, ${ }^{*} \mathrm{P}<0.05$ ).

of pregnancy-related diseases, such as PE (10). Although the apoptosis of trophoblastic cells increases in normal placentas with advancing gestation, increased apoptosis of trophoblastic cells has been observed in pregnancies complicated by PE $(11,12)$. The precise etiology of PE remains unclear; however, it may be associated with shallow trophoblastic invasion or impaired decidualization. In our previous study, we suggested that GABRP impaired remodeling of the decidua (7), and in the present study, we have demonstrated that GABRP promotes apoptosis and hence, inhibits the invasion of trophoblastic cells. Based on these findings, we hypothesized that GABRP may be a novel candidate which explains the association between the pathologies of the fetal placenta and maternal decidua in PE, and GABRP may adversely affect placental function through intrinsic (trophoblastic) factors in combination with extrinsic (maternal uterine) factors.

As important regulators of apoptosis, members of the Bcl-2 family may be divided into two functionally antagonistic groups: i) apoptosis suppressors such as $\mathrm{Bcl}-2, \mathrm{Bcl}-\mathrm{x}_{\mathrm{L}}$ and $\mathrm{Bcl}-\mathrm{w}$; and ii) apoptosis promoters such as $\mathrm{Bax}, \mathrm{Bcl}-\mathrm{x}_{\mathrm{S}}$, Bak and Bad (13-15). To identify the members of this protein family that may regulate trophoblast apoptosis, we assessed the expression of three important members of the Bcl-2 gene family in GABRP-overexpressing HTR-8/SVneo cells. As expected, the mRNA and protein expression of pro-apoptotic Bad and Bax was upregulated following GABRP overexpression. Unexpectedly, the anti-apoptotic Bcl-2 expression was also increased. It appears contradictory that GABRP activates both suppressors and promoters of apoptosis during the apoptosis cascade of HTR-8/SVneo cells. Even though it has been demonstrated that Bcl-2 may inhibit further propagation of the death signal at the mitochondrial level through mediating the activity of initiator caspases and the activation of execution caspases $(16,17)$, the increase in Bcl-2 following GABRP overexpression in the HTR-8/SVneo cells appears inadequate to prevent the whole apoptosis cascade when the 
A

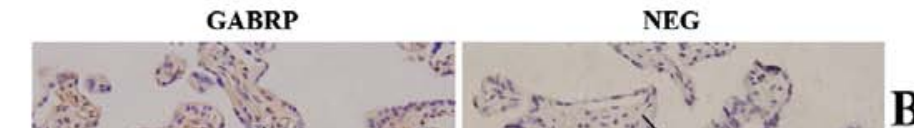

Control
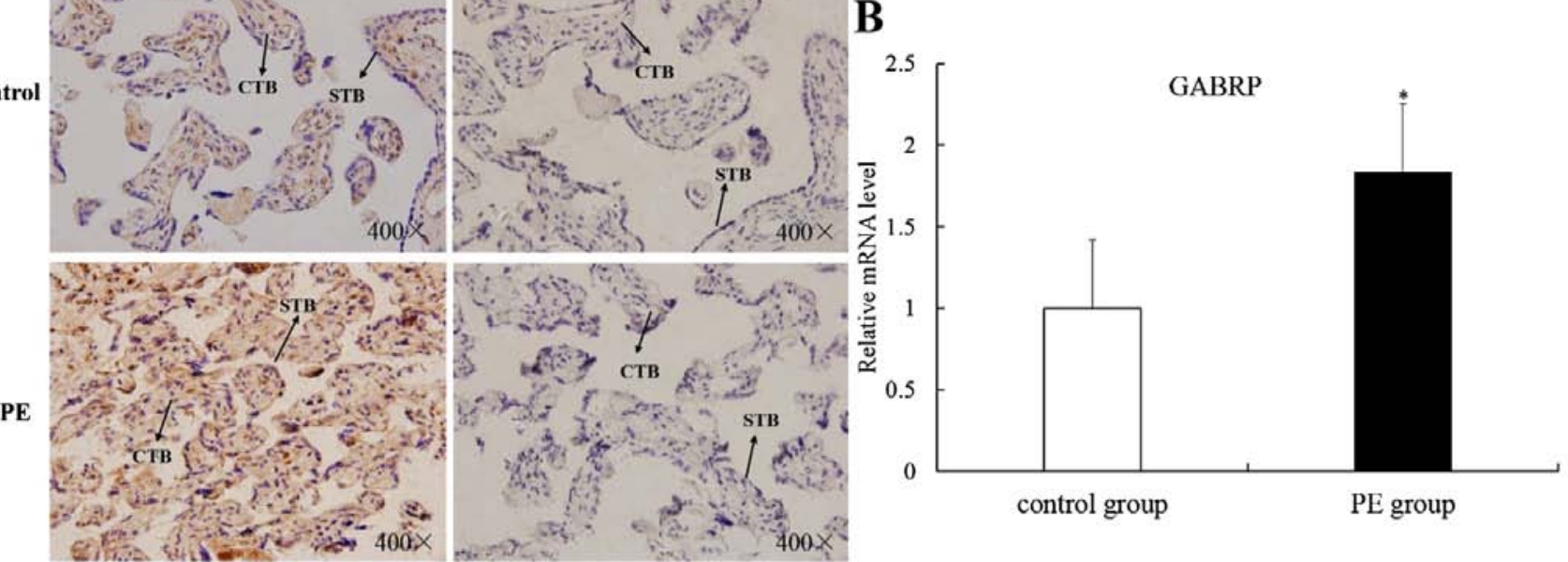

Figure 5. Differential expression of gamma-aminobutyric acid type A receptor $\pi$ subunit (GABRP) in placental tissues from patients with preeclampsia (PE) or normal pregnancy. (A) The immunostaining of GABRP was mainly localized in the cytotrophoblasts (CTBs) and was increased in the PE group compared with that in the control group. Negative placental tissues of the control group and the PE group are shown. NEG, negative controls in which normal IgG was used in place of primary antibody; STB, syncytiotrophoblast. (B) RT-qPCR revealed an increase in the mRNA level of GABRP in the PE group compared with that in the control group (t-test, $\left.{ }^{*} \mathrm{P}<0.05\right)$. Original magnification $\mathrm{x} 400$.
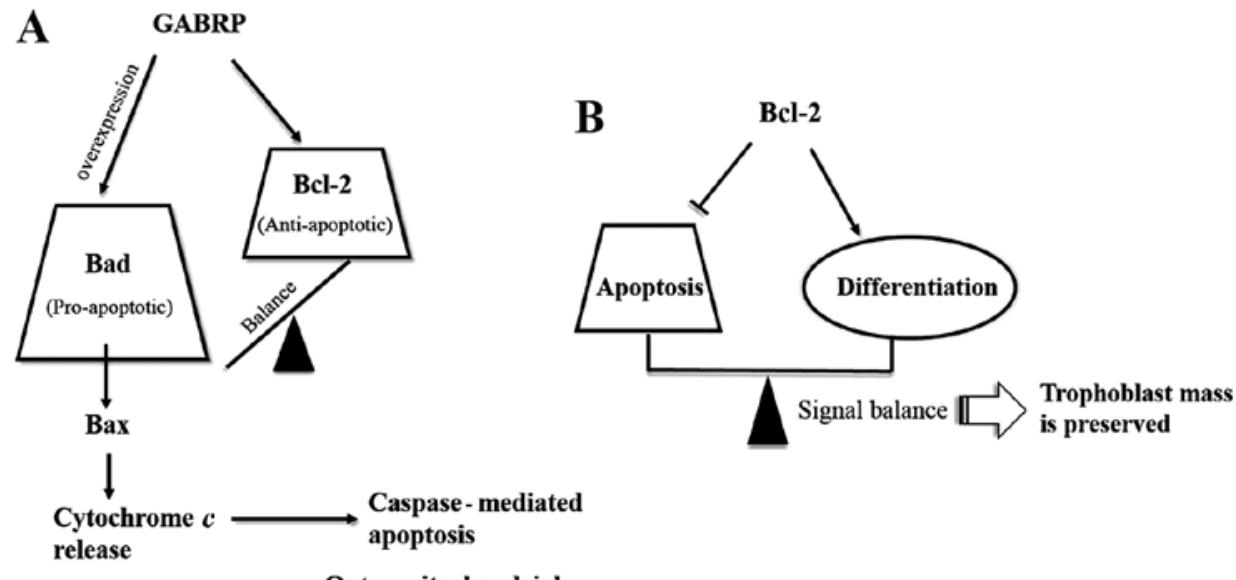

Outer mitochondrial

membrane

Figure 6. Schematic depicting the effect of gamma-aminobutyric acid type A receptor $\pi$ subunit (GABRP) on members of the Bcl-2 family in the apoptosis cascade and the role of Bcl-2 in balancing the apoptosis and differentiation process. (A) GABRP maintains the balance between pro-apoptotic Bad and anti-apoptotic Bcl-2 under normal conditions. However, the balance may be lost and biased towards the pro-apoptotic Bad pathway when GABRP is overexpressed. Thereafter, Bax is activated which causes cytochrome $c$ release and activation of the caspase-mediated apoptosis cascade. (B) There is a differentiation-dependent pattern of Bcl-2 expression in the placenta whereby a signal balance between apoptosis and differentiation is achieved and hence, trophoblast mass is preserved during pregnancy.

execution caspases, such as caspase-3 and its active form, cleaved caspase-3, were also activated. Thus, we hypothesized that GABRP maintains a state of equilibrium between anti-apoptotic Bcl-2 and pro-apoptotic Bad. However, excessive GABRP may damage this balance and induce apoptosis along the Bad pathway (Fig. 6A). In addition, as Bcl-2 expression is STB-specific during pregnancy (18-22), it is tempting to speculate that Bcl-2 is associated with the differentiation of trophoblasts. In addition, the treatment of JEG-3 cells with 8-Br-cAMP, which induces genes characteristic of STBs, was shown to raise Bcl-2 protein levels approximately 2 -fold (23). Thus, we speculate that: i) there is a differentiation-dependent pattern of Bcl-2 expression in the placenta, with the protein being expressed in the STBs throughout gestation and increased in JEG-3 choriocarcinoma cells following treatment with the differentiation inducing reagent, 8-Br-cAMP; and ii) Bcl-2 expression establishes a delicate balance between the differentiation and apoptosis of trophoblasts and this may be one mechanism through which trophoblast mass is preserved during pregnancy (Fig. 6B). In fact, there is evidence to suggest that syncytial fusion is directly or indirectly associated with apoptotic events (24), and $\mathrm{Bcl}-2$ is involved in the retardation of the apoptosis cascade in the execution stages. In the initiation stages of the apoptosis cascade, caspase- 8 is believed to be required for syncytial fusion $(25,26)$. Nevertheless, the presumption that GABRP may be involved in the differentiation of trophoblasts through the upregulation of Bcl-2 merits further investigation. 
In mammalian brains, the synaptic inhibition of neuronal activity is principally mediated by GABA at GABA A receptors, and functional GABA A receptors have also been detected in nonneuronal cells (1). Among 16 known GABA A receptor subunits, GABRP mRNA was confirmed to be highly expressed in the human uterus as well as in normal breast tissue by RT-qPCR (2). In fact, there have been many studies regarding the role of GABRP in uterine and breast tissues. For example, GABRP mRNA expression and membrane translocation were suggested to be regulated by HOXA10, which is likely to be involved in initiating and maintaining a receptive endometrium (5). Furthermore, the dynamic expression of GABRP mRNA throughout pregnancy is suggested to correlate with parturition (4). Despite the abundance of GABRP in breast tissue, the role of GABRP in breast tissue remains controversial according to the findings of different groups. For example, Zafrakas et al postulated that GABRP may exert a tumor-suppressing effects in breast tissue (2), whereas Sizemore et al suggested that GABRP stimulates basal-like breast cancer cell migration through the activation of extracellular-regulated kinase 1/2 (27) and the distinct results may be due to the heterogeneity of breast cancer. In addition to uterine and breast tissues, it has been demonstrated that GABRP is overexpressed in pancreatic tumors (28) and promotes pancreatic cancer growth through GABA stimulation (29).

Emerging evidence indicates that GABA acts as a tumor signaling molecule in peripheral non-neuronal cells (30-32). Given the role of GABRP in the uterus and some tumor tissues, it is important to consider the potential function of GABRP in the trophoblastic cells of the reproductive system, which share a similar capacity to migrate and invade surrounding tissues with malignant cells (33-35). Herein, we have demonstrated that GABRP promotes apoptosis and hence, inhibits the invasion of trophoblastic cells, which may be associated with the onset of PE. In addition, the upregulation of GABRP was detected in placental tissues obtained from patients with $\mathrm{PE}$ compared with that in normal control placental tissues which is consistent with our hypothesis. This suggests that GABRP may possibly be used as a tissue marker with diagnostic significance in PE. On the other hand, GABRP overexpression may contribute to the progression of PE and GABRP may be a promising molecular target for the development of new therapeutic strategies for PE.

\section{Acknowledgements}

The present study was supported by grants from the National Natural Science Foundation of China (no. 31171436).

\section{References}

1. Hedblom E and Kirkness EF: A novel class of GABAA receptor subunit in tissues of the reproductive system. J Biol Chem 272: 15346-15350, 1997.

2. Zafrakas M, Chorovicer M, Klaman I, Kristiansen G, Wild PJ, Heindrichs U, Knüchel R and Dahl E: Systematic characterisation of GABRP expression in sporadic breast cancer and normal breast tissue. Int J Cancer 118: 1453-1459, 2006.

3. Gladkevich A, Korf J, Hakobyan VP and Melkonyan KV: The peripheral GABAergic system as a target in endocrine disorders. 124: 1-8, 2006.

4. Fujii E and Mellon SH: Regulation of uterine gamma-aminobutyric acid(A) receptor subunit expression throughout pregnancy. Endocrinology 142: 1770-1777, 2001.
5. Sadeghi H and Taylor HS: HOXA10 regulates endometrial GABAA (pi) receptor expression and membrane translocation. Am J Physiol Endocrinol Metab 298: E889-E893, 2010.

6. Longtine MS, Chen B, Odibo AO, Zhong Y and Nelson DM: Villous trophoblast apoptosis is elevated and restricted to cytotrophoblasts in pregnancies complicated by preeclampsia, IUGR, or preeclampsia with IUGR. Placenta 33: 352-359, 2012.

7. Luo W, Liu Z, Tan D, Zhang Q, Peng H, Wang Y and Tan Y: Gamma-amino butyric acid and the A-type receptor suppress decidualization of mouse uterine stromal cells by down-regulating cyclin D3. Mol Reprod Dev 80: 59-69, 2013.

8. Allaire AD, Ballenger KA, Wells SR, McMahon MJ and Lessey BA: Placental apoptosis in preeclampsia. Obstet Gynecol 96: 271-276, 2000.

9. Zhang Q, Chen Q, Lu X, Zhou Z, Zhang H, Lin HY, Duan E, Zhu C Tan Y and Wang H: CUL1 promotes trophoblast cell invasion at the maternal-fetal interface. Cell Death Dis 4: e502, 2013.

10. Sharp AN, Heazell AE, Crocker IP and Mor G: Placental apoptosis in health and disease. Am J Reprod Immunol 64: 159-169, 2010.

11. Straszewski-Chavez SL, Abrahams VM and Mor G: The role of apoptosis in the regulation of trophoblast survival and differentiation during pregnancy. Endocr Rev 26: 877-897, 2005.

12. Crocker IP, Cooper S, Ong SC and Baker PN: Differences in apoptotic susceptibility of cytotrophoblasts and syncytiotrophoblasts in normal pregnancy to those complicated with preeclampsia and intrauterine growth restriction. Am J Pathol 162: 637-643, 2003.

13. Marzioni D, Mühlhauser J, Crescimanno C, Banita M, Pierleoni C and Castellucci M: BCL-2 expression in the human placenta and its correlation with fibrin deposits. Hum Reprod 13: 1717-1722, 1998.

14. Krajewski S, Krajewska M and Reed JC: Immunohistochemical analysis of in vivo patterns of Bak expression, a proapoptotic member of the Bcl-2 protein family. Cancer Res 56: 2849-2855, 1996.

15. Cory S, Huang DC and Adams JM: The Bcl-2 family: roles in cell survival and oncogenesis. Oncogene 22: 8590-8607, 2003.

16. Huppertz B and Kingdom JC: Apoptosis in the trophoblast - role of apoptosis in placental morphogenesis. J Soc Gynecol Investig 11: 353-362, 2004.

17. Huppertz B, Kadyrov M and Kingdom JC: Apoptosis and its role in the trophoblast. Am J Obstet Gynecol 195: 29-39, 2006.

18. Ratts VS, Tao XJ, Webster CB, Swanson PE, Smith SD, Brownbill P, Krajewski S, Reed JC, Tilly JL and Nelson DM: Expression of BCL-2, BAX and BAK in the trophoblast layer of the term human placenta: a unique model of apoptosis within a syncytium. Placenta 21: 361-366, 2000.

19. Murakoshi H, Matsuo H, Laoag-Fernandez JB, Samoto T and Maruo T: Expression of Fas/Fas-ligand, Bcl-2 protein and apoptosis in extravillous trophoblast along invasion to the decidua in human term placenta. Endocr J 50: 199-207, 2003

20. Ho S, Winkler-Lowen B, Morrish DW, Dakour J, Li H and Guilbert LJ: The role of Bcl-2 expression in EGF inhibition of TNF-alpha/IFN-gamma-induced villous trophoblast apoptosis. Placenta 20: 423-430, 1999.

21. Candelier JJ, Frappart L, Yadaden T, Poaty H, Picard JY, Prévot S and Coullin P: Altered p16 and Bcl-2 expression reflects pathologic development in hydatidiform moles and choriocarcinoma. Pathol Oncol Res 19: 217-227, 2013.

22. Lea RG, al-Sharekh N, Tulppala M and Critchley HO: The immunolocalization of bcl-2 at the maternal-fetal interface in healthy and failing pregnancies. Hum Reprod 12: 153-158, 1997.

23. Sakuragi N, Matsuo H, Coukos G, Furth EE, Bronner MP, VanArsdale CM, Krajewsky S, Reed JC and Strauss JF III: Differentiation-dependent expression of the BCL-2 proto-oncogene in the human trophoblast lineage. J Soc Gynecol Investig 1: 164-172, 1994.

24. Huppertz B, Tews DS and Kaufmann P: Apoptosis and syncytial fusion in human placental trophoblast and skeletal muscle. Int Rev Cytol 205: 215-253, 2001.

25. Black S, Kadyrov M, Kaufmann P, Ugele B, Emans N and Huppertz B: Syncytial fusion of human trophoblast depends on caspase 8. Cell Death Differ 11: 90-98, 2004.

26. Gauster M, Siwetz M, Orendi K, Moser G, Desoye G and Huppertz B: Caspases rather than calpains mediate remodelling of the fodrin skeleton during human placental trophoblast fusion. Cell Death Differ 17: 336-345, 2010.

27. Sizemore GM, Sizemore ST, Seachrist DD and Keri RA: GABA(A) receptor pi (GABRP) stimulates basal-like breast cancer cell migration through activation of extracellular-regulated kinase 1/2 (ERK1/2). J Biol Chem 289: 24102-24113, 2014. 
28. Johnson SK and Haun RS: The gamma-aminobutyric acid A receptor pi subunit is overexpressed in pancreatic adenocarcinomas. JOP 6: 136-142, 2005.

29. Takehara A, Hosokawa M, Eguchi H, Ohigashi H, Ishikawa O, Nakamura Y and Nakagawa H: Gamma-aminobutyric acid (GABA) stimulates pancreatic cancer growth through overexpressing GABAA receptor pi subunit. Cancer Res 67: 9704-9712, 2007.

30. Young SZ and Bordey A: GABA's control of stem and cancer cell proliferation in adult neural and peripheral niches. Physiology (Bethesda) 24: 171-185, 2009.

31. Watanabe M, Maemura K, Oki K, Shiraishi N, Shibayama Y and Katsu K: Gamma-aminobutyric acid (GABA) and cell proliferation: focus on cancer cells. Histol Histopathol 21: 1135-1141, 2006.

32. Ortega A: A new role for GABA: inhibition of tumor cell migration. Trends Pharmacol Sci 24: 151-154, 2003.
33. Perry JK, Lins RJ, Lobie PE and Mitchell MD: Regulation of invasive growth: similar epigenetic mechanisms underpin tumour progression and implantation in human pregnancy. Clin Sci (Lond) 118: 451-457, 2009.

34. Murray MJ and Lessey BA: Embryo implantation and tumor metastasis: common pathways of invasion and angiogenesis. Semin Reprod Endocrinol 17: 275-290, 1999.

35. Ferretti C, Bruni L, Dangles-Marie V, Pecking AP and Bellet D: Molecular circuits shared by placental and cancer cells, and their implications in the proliferative, invasive and migratory capacities of trophoblasts. Hum Reprod Update 13: 121-141, 2007. 